Document downloaded from:

http://hdl.handle.net/10251/57093

This paper must be cited as:

Ruiz Fernández, LA.; Recio Recio, JA.; Fernández-Sarría, A.; Hermosilla, T. (2011). A feature extraction software tool for agricultural object-based image analysis. Computers and Electronics in Agriculture. 76(2):284-296. doi:10.1016/j.compag.2011.02.007.

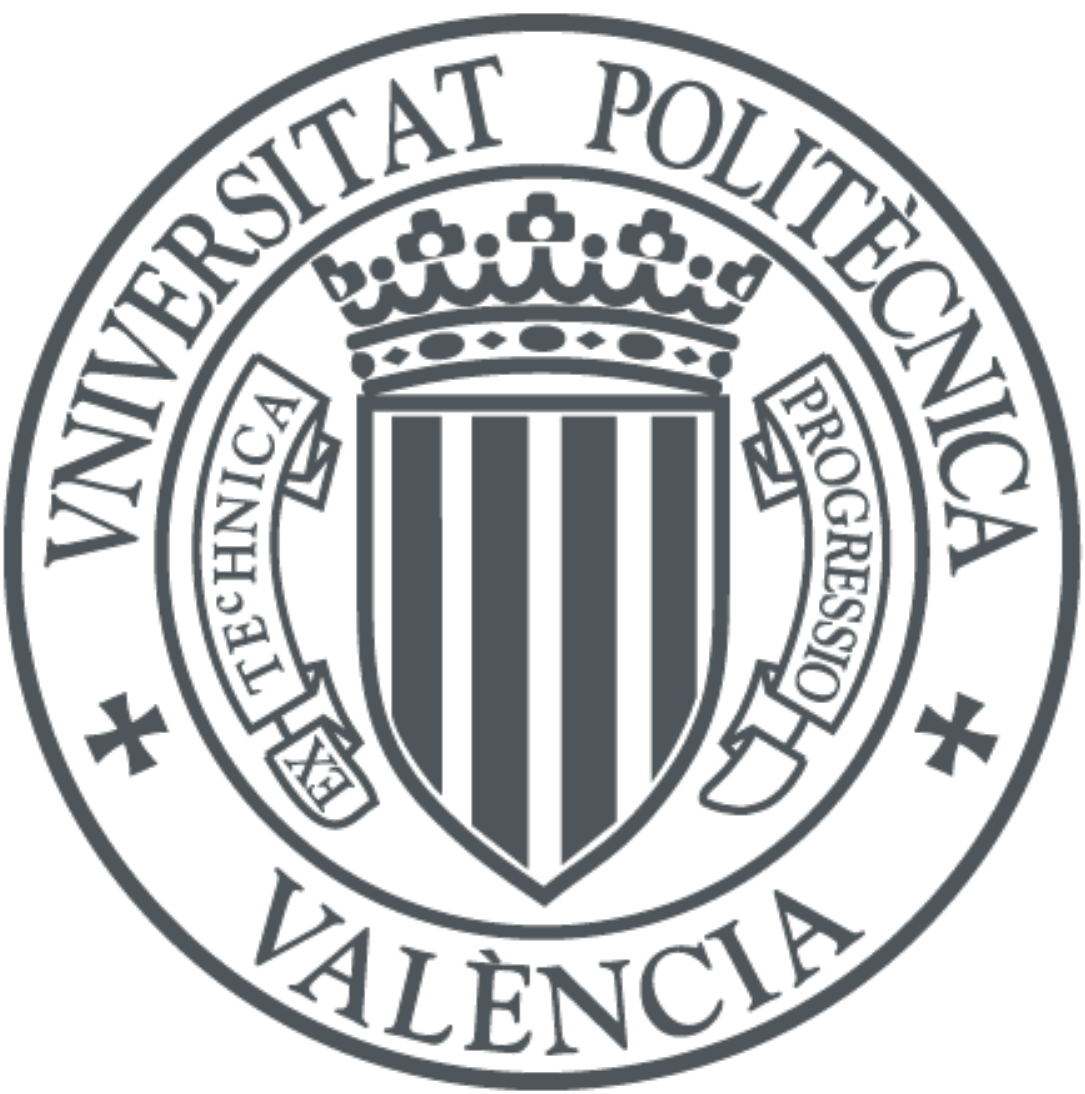

The final publication is available at

http://dx.doi.org/10.1016/j.compag.2011.02.007

Copyright Elsevier

Additional Information 


\title{
A FEATURE EXTRACTION SOFTWARE TOOL FOR AGRICULTURAL OBJECT-BASED IMAGE ANALYSIS
}

\author{
L.A. Ruiz, J.A. Recio, A. Fernández-Sarría, T. Hermosilla.
}

Departamento de Ingeniería Cartográfica, Geodesia y Fotogrametría, Universidad Politécnica de Valencia, Camino de Vera, s/n, 46022 Valencia, Spain

Corresponding author: Luis Ángel Ruiz

e-mail address: laruiz@cgf.upv.es

Postal address: Departamento de Ingeniería Cartográfica, Geodesia y Fotogrametría Universidad Politécnica de Valencia

Camino de Vera, s/n. 46022 Valencia. SPAIN

Telephone: $\quad 0034963877000$ ext. 75536

Fax Number: 0034963877559.

\section{ABSTRACT}

A software application for automatic descriptive feature extraction from image-objects, FETEX 2.0, is presented and described in this paper. The input data include a multispectral high resolution digital image and a vector file in shapefile format containing the polygons or objects, usually extracted from a geospatial database. The design of the available descriptive features or attributes has been mainly focused on the description of agricultural parcels, providing a variety of information: spectral information from the different image bands; textural descriptors of the distribution of the intensity values based on the grey level co-occurrence matrix, the wavelet transform and a factor of edgeness; structural features describing the spatial arrangement of the elements inside the objects, based on the semivariogram curve and the Hough transform; and several descriptors of the object shape. The output file is a table that can be produced in four alternative formats, containing a vector of features for every object processed. This table of numeric values describing the objects from different points of view can be externally used as input data for any classification software. Additionally, several types of graphs and images 
describing the feature extraction procedure are produced, useful for interpretation and understanding the process. A test of the processing times is included, as well as an application of the program in a real parcel-based classification problem, providing some results and analyzing the applicability, the future improvement of the methodologies, and the use of additional types of data sets. This software is intended to be a dynamic tool, integrating further data and feature extraction algorithms for the progressive improvement of land use/land cover database classification and agricultural database updating processes.

Keywords: feature extraction, parcel-based, image analysis, remote sensing, agricultural database updating, semivariogram, Hough transform, texture.

\section{1.- INTRODUCTION}

Image classification techniques are frequently used in remote sensing to face a wide range of applications, being traditionally focused on the analysis of independent pixels using multiple spectral bands as input data, sometimes introducing additional information related to the spatial distribution of the intensity values of the neighbourhood of the pixels, such as the case of the texture based approaches. Much attention has been paid to the process of creating the appropriate decision functions that optimize the accuracy results, and many different methodologies for the classification itself have been tested. However, a crucial step to be taken before the classification is the synthesis of the information or attributes that describe the image element to be classified. This is usually called the feature extraction process, and much less effort has been made in this sense, mainly because the classification has been fundamentally pixel-based, and not object-based, thus restricting the options used to enrich the set of features used as input for the classifiers. 

Geographic Information Science devoted to partitioning remote sensing imagery into meaningful image-objects, and assessing their characteristics through spatial, spectral and temporal scale (Hay and Castilla, 2006). Before carrying out feature extraction and classification steps, these techniques require image segmentation. Segmentation refers to the process of partitioning a digital image into multiple segments, called image-objects or simply objects, in order to simplify and/or change the representation of an image into a more meaningful and homogeneous structure that is easier to analyze (Shapiro and Stockman, 2001). These segments have additional spectral and spatial information when compared with single pixels (Blaschke, 2010). Segmentation is the main problem of this analysis of the image because it has multiple solutions (Hay and Castilla, 2006; Zhang et al., 2008). Depending on the method and the parameters used, the results, that are the objects created, can substantially change (Meinel and Neubert, 2004; Neubert and Herold, 2008; Smith and Morton, 2008; Van Coillie et al., 2008). Differing from the automatic image segmentation algorithms, objects can be created using the cartographic limits contained in spatial geodatabases. This approach is known as Parcel-Based Image Analysis and has some advantages regarding to other image analysis techniques, the most important being the possibility to directly link the information of the image to the information contained in a database (Berberoglu and Curran, 2004; Walter, 2004). Moreover, for many land use mapping applications, parcel-based classification has been reported to be more accurate than pixel-based classification (Pedley and Curran, 1991; Janssen and Molenaar, 1995; Aplin et al., 1999; Berberoglu et al., 2000; Volante et al., 2007).

Once object definition is resolved, the next step is to accurately describe each group of pixels in order to facilitate the correct classification of the object. Different commercial software tools have been made available over the last several years, providing a variety of features to describe the properties of the objects and their mutual relations. These features can be grouped into spectral, textural, shape, thematic attributes, relative to other objects in the same or different segmentation levels and relative to the global image (Baatz et al., 2004). Our need for a tool to 
automate the descriptive feature extraction process, allowing us to design and incorporate new sets of features describing objects from different perspectives, evaluating and comparing their performance, led us to develop FETEX 2.0, an interactive computer program for image objectoriented feature extraction. The software was written in IDL 6.2 and it can be used on ENVI 4.2 or higher. Our aim with this program is to develop new object descriptive features, some of them not included in commercial software tools, designed to characterize specific types of parcels, and with the capacity to adapt certain computation parameters to particular problems. These features can eventually be used with any classifier in order to assign a class to each object. The software requires, as input data, images and vectorial files containing the polygon boundaries in ESRI shapefile (shp) format.

FETEX 2.0 does not include any segmentation algorithm, but it creates the objects according to the limits contained in the vectorial file. From each one of the objects created, spectral, textural, shape and structural features can be extracted. The structural features provide information about the distribution of elements inside each object, detecting and quantifying possible spatial patterns, very often relevant in identifying the land use/land cover of objects, especially when working in agricultural areas. For each object, the output is a feature vector ready to be used with the selected classifier. Information obtained with FETEX 2.0 can be used not only in the classification of the database objects, but also as ancillary information in agricultural inventories. Such ancillary information can be the number of trees in a parcel, their average size, planting distances, etc. Additional images and explanatory graphs about the features extracted that may be useful for interpretation of results can be obtained as well.

FETEX 2.0 is designed to work with land use/land cover databases in order to help in the process of classification of the existing parcels, or also to determine the changes that have occurred in a database comparing the current classified image with the old database. It has been applied successfully in the feature extraction phase in several updating land use/land cover cartography processes (Ruiz et al., 2009). Interested users are most likely to be among the cartographic research community and official cartographical institutions devoted to updating 
and maintaining large land use/land cover databases. A limited version of FETEX 2.0 is available at the Geo-Environmental Cartography and Remote Sensing Research Group (CGAT) website (http://cgat.webs.upv.es).

\section{2.- EXTRACTION OF DESCRIPTIVE FEATURES WITH FETEX 2.0}

The main reason for developing the program was to create a tool for supporting the process of classification and updating of land use/land cover spatial databases from an object-based perspective. The extraction of valuable features for this process is essential. FETEX 2.0 is designed to independently process each image-object to extract a variety of descriptive features useful to characterize the current land use/land cover. These features can be grouped into five categories: spectral, textural, structural, shape and those extracted from ancillary data. In this section, the features are briefly described or referenced.

\section{1.- Spectral features}

Spectral features provide information about the spectral response of objects, which depends on land coverage types, state of vegetation, soil composition, construction materials, etc. These features are especially useful in the characterization of spectrally homogeneous objects, such as herbaceous crops, fallow fields or compact industrial areas. This group of features constitutes the traditional information derived from any type of multispectral imagery. In addition to the original spectral bands, any combination, ratio, or transformation (principal components, tasselled cap bands, etc) can be included as complementary bands in the input raster file to be processed.

For each band contained in the input raster file, the values of mean, standard deviation, minimum, maximum, range, sum and majority of the pixel values inside each object can be calculated.

\section{2.- Texture features}


131 The texture informs about the spatial distribution of the intensity values in the image, providing

132 information about contrast, uniformity, rugosity, regularity, etc. A considerable number of 133 quantitative texture features and approaches have been reported using different methodologies.

134 Traditionally, they are computed considering the neighbourhood of each pixel on the image. In our case, each texture feature value is referred to a particular object, since it is extracted from each group of pixels that constitute an object. The simplest manner to characterize texture is

137 based on the first order histogram features. Features such as kurtosis and skewness, representing 138 the distribution of values of the histogram of an object, are included in FETEX 2.0. The most widely used set of features is that proposed by Haralick et al. (1973), based on the grey level cooccurence matrix (GLCM) and also called second order histogram features. Up to seven of these features can be extracted by FETEX 2.0: contrast, uniformity, entropy, variance, covariance or product moment, inverse difference moment, and correlation. Since an object-oriented approach is used, only one GLCM is computed for each object, describing the co-occurrences of the pixel values that are separated at a distance of one pixel inside the polygon, and considering the average value of four principal orientations $\left(0^{\circ}, 45^{\circ}, 90^{\circ}\right.$ and $\left.135^{\circ}\right)$ in order to avoid the influence of the orientation of the elements inside the objects, keeping in mind a potential classification process. Therefore, only one value for every GLCM feature is computed for each object. Although the mean of the GLCM is one of the most widely used features in texture image classification problems at the pixel level, due to its very high correlation to the mean value of the original band, this feature has been intentionally excluded from the set of GLCM

151 features.

152 Another powerful feature is the edgeness factor, that represents the density of edges present in a 153 neighbourhood. Sutton and Hall (1972) proposed the following formula to compute the 154 edgeness factor $\mathrm{g}$ :

$$
g(d)=\sum_{(i, j) \in N}\{|I(i, j)-I(i+d, j)|+|I(i, j)-I(i-d, j)|+|I(i, j)-I(i, j+d)|+|I(i, j)-I(i, j-d)|\}
$$


where $g$ is computed as a function of the distance $d$ between pixels of an image $I$ in a neighbourhood $N$. Due to the good performance of this feature in different landscape classification problems (Ruiz et al., 2002; 2004), it has been included in the program, and the mean and the standard deviation of the edgeness factor for each parcel is computed.

\section{3.- Wavelet-based texture features}

Although the basic ideas of wavelets existed since the beginning of last century, the applied mathematical models were developed in the mid-eighties. A review and mathematical description of wavelets can be found in Bultheel (1995), and Walker (1999). The use of the wavelet transform for texture analysis was first proposed by Mallat (1989). Since the texture of an image is a function of the scale, an advantage of wavelet decomposition is that it provides a unified framework for multiscale analysis. The wavelet transform allows for the decomposition of a signal using a series of elemental functions called wavelets and scaling, which are created by the scaling and translation of a base function, known as the mother wavelet:

$$
\psi_{s, u}=\frac{1}{\sqrt{s}} \psi\left(\frac{x-u}{s}\right), s \in \mathfrak{R}^{+}, u \in \mathfrak{R}
$$

where $s$ governs the scaling and $u$ the translation. The wavelet decomposition of a function is obtained by applying each of the elemental functions or wavelets to the original function:

$$
W f(s, u)=\int_{\mathfrak{R}} f(x) \frac{1}{\sqrt{s}} \psi^{*}\left(\frac{x-u}{s}\right) d x
$$

In the practice of image analysis, the extension to a 2-D discrete function is usually performed by means of a product of 1-D low-pass and high-pass filters. As a result, the wavelet transform decomposes the original image into a series of images with different scales, called trends and fluctuations. The former are averaged versions of the original image, and the latter contain the high frequencies at different scales or levels. Since the most relevant texture information is lost in the lowpass filtering process, only fluctuations are used to calculate texture descriptors. If the 
inverse transform is applied to the fluctuations, three reconstructed images, or details, are

177 obtained: horizontal, vertical and diagonal. This process is called multiresolution analysis.

178 Different texture features have been extracted from wavelet details or fluctuations, such as the 179 local energy (Randen and Husoy, 1999), variance filter (Ferro and Warner, 2002), histogram 180 signatures (Simard et al., 1999), or co-occurrence features (Van de Wouwer et al., 1999; Ruiz et 181 al., 2004).

182 The application FETEX 2.0 includes some texture features based on the wavelet transform. 183 Seven families of wavelet functions (Haar, Daubechies, Coiflet, Meyer, Symlet, Shannon and Battle-Lemarié) can be applied over the image objects. Defining the support of a wavelet function as the smallest closed interval, outside of which the function is zero (Bultheel, 1995), different supports have been defined for each wavelet family, following the work of FernándezSarría (2007). A total of eight Haralick's features derived from the GLCM can be extracted from the image containing the sum of the reconstructed details (mean, contrast, uniformity, entropy, variance, covariance, inverse difference moment, and correlation), as well as the mean and standard deviation of the edgeness factor.

However, applying the wavelet transform using an object-oriented approach may be a problem when large supports are used, since a higher proportion of neighbour pixels located outside the analyzed object will be considered in the transformation process. Two measures are followed to reduce this effect: First, an erosion filter using a circular structuring element with a diameter size equal to the support of the wavelet function is applied to the final image. A limitation of this is that small and/or narrow objects will be completely eroded, and subsequently omitted from the characterization of the features that belong to this group. Secondly, even if up to three levels of decomposition are included for computation in FETEX, only the first level is enabled in the current version, in order to avoid the decimation of the image to the point that the object practically dissapears when the direct transform is applied. This effect will be negligible when working with large objects. 
203

204

205

206

207

208

209

210

211

212

Structural features provide information of the spatial arrangement of different elements in the object, in terms of randomness or regularity of the distribution of the elements. This is the case of alignments or regular patterns that are present in different man-made lanscapes, such as the planting patterns of crops and trees in agricultural plots (Recio et al., 2006; Ruiz et al., 2007). The identification of regular planting patterns can be particularly useful in agricultural classification, as reported by several authors (Trías-Sanz, 2006; Helmholz et al., 2007; Ruiz et al., 2009; Recio, 2009; Hermosilla et al., 2010). Structural features computed in FETEX 2.0 are divided into two groups: the semivariogram and the Hough transform derived features.

\subsection{1.- Features extracted from the experimental semivariogram}

The semivariogram quantifies the spatial associations of the values of a variable, and measures the degree of spatial correlation between different pixels in an image. This is a particularly suitable tool in the characterization of regular patterns. The expression which describes the experimental semivariogram of a continuous variable is:

$$
\gamma(h)=\frac{1}{2 N} \sum_{i=1}^{N}\left[z\left(x_{i}\right)-z\left(x_{i}+h\right)\right]^{2}
$$

where $z\left(x_{i}\right)$ represents the value of the variable in position $x_{i}, N$ is the number of pairs of data considered and $h$ provides the separation between elements in a given direction.

The semivariogram has been widely employed in digital image processing. Its usefulness in remote sensing has been demonstrated, complementing the spectral variables with information related to the spatial structure of the image (Carr, 1996; Durrieu et al., 2005). The relationship between the range of the semivariogram and the size of the pattern described by the objects of an image has been studied by Woodcock et al. (1988a, 1988b). Carr and Miranda (1998) used the slope in the origin as a feature directly related to the variability of the intensity values in these objects. Other works are focused on the extraction of descriptive features from the 
semivariogram of remotely sensed images. Thus, Chica-Olmo and Abarca-Hernandez (2000) computed the first value of that function in the neighbourhood of a pixel to characterize the texture of that neighbourhood. Maillard (2003) used all the semivariogram values, conferring more importance to the initial values, and less to the subsequent values. These and other authors (Jakomulska and Clarke, 2000; Ashoori et al., 2008; etc.) used different features extracted from the semivariogram computed in a window around a pixel in order to perform a classification.

The omnidirectional semivariogram can be obtained by averaging the semivariograms of all possible directions. However, this approach requires a long processing time. This semivariogram is obtained in FETEX 2.0 by computing, for each object, the mean of the semivariograms calculated in six different directions, ranging from $0^{\circ}$ to $150^{\circ}$ with a step of $30^{\circ}$. Afterwards, each semivariogram curve is smoothed using a Gaussian filter with a stencil of 3 positions, in order to reduce experimental fluctuations.

In homogeneous objects, semivariance values tend to be higher as the lag increases. However, when the elements inside an object are spatially arranged following a regular pattern, the semivariogram has a cyclic behaviour, and it is known as hole-effect semivariogram (Pyrcz and Deutsch, 2003). This type of behaviour is common in areas with a high level of human intervention, such as certain crops, urban or industrial landscapes. Figure 1 shows the experimental semivariogram curves of four parcels with different land uses. Figure 1a and Figure 1c present parcels containing tree crops that follow regular plantation patterns, their semivariograms being examples of hole-effect semivariograms. On the other hand, Figure $1 \mathrm{~b}$ and Figure 1d do not present regular patterns or spatial cyclicity, and their semivariogram curves follow a monotonous rising trend.

247 The features extracted by FETEX 2.0 are based on the zonal analysis defined by a set of 248 singular points on the semivariogram, such as the first maximum, the first minimum, the second maximum, etc., and are fully described in Balaguer et al. (2010). The semivariogram derived features are: ratio variance at first lag, ratio between semivariance values at second and first 
lag, first derivative near the origin, lag value corresponding to the first maximum, mean of the semivariogram values up to the first maximum, variance of the semivariogram values up to the first maximum, area between the semivariogram value in the first lag and the semivariogram function until the first maximum, ratio between the semivariance at first local maximum and the mean semivariogram values up to this maximum, distance between the location of the first local maximum and the second local maximum, and distance between the first maximum and the first minimum.

In terms of efficiency in processing time, instead of computing the semivariogram considering all the pixels inside each object, only a random selection of pixels is used, in order to reduce the processing time. A test was carried out in order to assess the influence of the percentage of pixels used in two processes: in the calculation of the semivariogram curve, and in the performance of the classification of a sample of $N$ objects. The results show that semivariograms calculated using a reduced number of random pixels are very similar to those computed using all the pixels inside each object, as shown in the two examples of Figure 2. accuracies obtained in a per-object classification using the set of features derived from the semivariogram described above. Ten classification processes have been compared. In each, a different percentage of pixels has been randomly selected for semivariogram calculation. The overall accuracy obtained using $100 \%$ of the pixels is $81.3 \%$. The results reveal that the semivariogram derived features computed when using only $15 \%$ of the pixels describe the objects with an efficiency similar to that of when all the pixels are used, since they do not produce a significant reduction in the classification accuracy. Besides, the processing time is linearly reduced: $85 \%$ using only $15 \%$ of pixels, $75 \%$ considering a $25 \%$ and so on.

\subsection{2.- Features derived from the Hough transform}

The planting pattern and planting distances are key factors employed by photointerpreters to distinguish different crop typologies. Thus, once the information about global regularity of the 
277 parcel is extracted from the semivariogram analysis, the plantation pattern can be more 278 profoundly analysed in order to obtain more specific descriptors that complement the semivariogram derived features. For this purpose, a variety of features based on the Hough transform are included in FETEX 2.0.

281 The first step in the extraction of these features is the location of trees, which is done using the local maximum filtering (LMF) method (Gourgeon, 1999) from high spatial resolution imagery. The LMF is based on the assumption that reflectance is highest at the tree apex and decreases towards the crown edge (Wulder et al., 2000). Moving a kernel over the image, trees are found when the central value in the kernel window is higher than all other values. The scene illumination has an important influence on local maxima position, displacing their position from the real apex location. However, this displacement has no negative effects because it equally affects all the maxima.

LMF method is applied over NDVI images using a circular kernel with variable size to detect adult trees. This size is automatically determined for each object by the position of the first maximum on the semivariogram curve, being constrained by the interval defined between two thresholds that are set by the user. If the first maximum is lower than the lower threshold, this threshold will be used as the diameter of the kernel. In a similar way, if the first maximum is greater than the upper threshold, the diameter of the kernel will be the upper threshold. Assuming a regular distribution of trees in a parcel, the kernel diameter is related to the average size of the trees contained in a parcel.

Most of these features are designed for the classification of agricultural tree orchards. In order to facilitate this task, two main groups of trees can be considered: adult trees, having a considerable canopy, and young trees, with almost no vegetation cover. A threshold defined over the NDVI image is selected in order to distinguish those two groups of parcels. In those parcels with a NDVI mean value lower than the defined threshold, instead of applying LMF 
infrared (IR) band. This variation is used in order to locate the young trees recently planted. The result is a binary image where each located tree is represented by a pixel. In both cases, adult and young trees, pixel location must accomplish an additional condition: the maximum in NDVI band must be greater than a threshold fixed by the user and the minimum in IR band must be lower than a threshold, which must be empirically defined depending on the image characteristics. From this binary image, main tree alignments are extracted and characterized applying the Hough transform (Hough, 1962).

The Hough transform is a method that can be used in image processing to locate curves that can be parameterized as straight lines, polynomials or circles. This method has been widely used on images for row detection in agricultural crops (Reid and Searcy, 1986; Leemans and Destain, 2006; Gée et al., 2008). Structural information derived from the Hough transform has also been used for automatic classification and characterization of agricultural landscapes. Chanussot et al. (2005) applied the Fourier transform over a vineyard image, and then, the Hough transform for estimating and representing the crop orientation. Trías-Sanz (2006) employed structural properties based on orientation features to discriminate between different vegetation covers. He applied the variogram transformation and then, over the resultant image, the Hough transform, obtaining the orientation histogram. Helmholz et al. (2007) used the orientation information directly derived from the Hough transform to separate between tilled and untilled plots based on the existence of a minimum number of parallel lines.

The principle of the Hough transform is based on the fact that an infinite number of straight lines can go through a single point of the plane. The purpose of the method is to determine which of these theoretical lines go through more points in the image, that is, to find the best fitting lines to the set of points that are present in the image. The method is based on the transformation of the coordinates from a Cartesian image space to a polar coordinate space. A point in the Cartesian space corresponds to a sinusoid in the polar space, representing the parameters of the lines passing through that point. A line in the Cartesian space is defined by the intersection of two or more sinusoids in the polar space. 
330 After thresholding the polar space to remove lines passing through a small number of points,

331 remaining lines are grouped into a histogram of frequencies for all directions ranging from $0^{\circ}$ to

$332180^{\circ}$. When some regularity in their spatial arrangement exists, two histogram maxima appear, 333 corresponding to the principal directions or alignments of trees in the parcel. Figure 4 shows an example of this: a tree parcel (Figure 4a); the binary image with the local maxima representing the location of trees, with the orientations of the two principal alignments superimposed (Figure 4b); the result of the Hough transform (Figure 4c). The existence of points where several curves converge indicates the presence of alignments in the Cartesian domain. Grouping these values in a histogram of frequencies, the two orientations of the principal alignments in the parcel are easily differentiated at $75^{\circ}$ and $161^{\circ}$ (Figure 4d). By isolating these orientations, the angular difference between them provides information about the orthogonality of the alignments. The distance between the points where the curves converge on the same direction in the Hough

342 domain correspond to the distance between the lines following that direction. These distances are used to describe the planting pattern size of tree crops along the two main orientations

\section{4 (Figure 5).}

345 A set of additional features related to the regularity in the distribution of trees are extracted from 346 this transformation and the histogram of orientations. These features are: proportion of points 347 included in the principal and secondary direction with respect to the total amount of points; mean, median and standard deviation of the distances between straight lines in the principal and secondary directions; proportion of straight lines in the principal and secondary directions; and angular difference between the two principal directions.

\section{5.- Shape features}

352 The shape features computed in FETEX 2.0 inform about the shape complexity of the objects, 353 and are mainly based on ratios between the area and the perimeter of the objects. These 354 descriptors, extracted directly from the geometric definition of the polygons contained in the 
database (parcels) can help to distinguish and identify different elements with particular shapes, such as roads, rivers, circular plots, etc.

The features available in the application are: compactness (Bogaert et al., 2000), shape index and fractal dimension (Krummel et al., 1987; McGarigal and Marks, 1995) (see Table 1). The area and perimeter of each object are also computed.

\section{6.- Ancillary data}

Depending on the algorithm used in a subsequent classification process, discrete variables can be included as descriptive features. Some studies (Rogan et al., 2003; Recio et al., in press) have shown that the combination of the historical land use of the parcels contained in an old database with spectral features may increase the overall accuracy of the classification. Some other discrete information, such as soil type and composition (Huang and Jensen, 1997), irrigation type, etc. can sometimes be useful to better describe the parcels or polygons. If this information is included in the spatial geodatabase as an input in FETEX 2.0, it can also be added as an output to the descriptive feature vector of each parcel.

Any other georreferenced information in raster format can also be added as extra input to extract information and characterize the objects. Thus, digital terrain models (elevation, slope or aspect) (Hoffer, 1975; Hutchinson, 1982), distance maps (Debeir et al., 2002; Mas, 2003; Recio et al., 2010), results of a per-pixel classification (Recio, 2009) or others, can be added as additional bands in order to compute statistics to describe the parcels.

\section{3.- THE PROGRAM}

\section{1.- Graphic User Interface}

The Graphic user interface of FETEX 2.0 is a window menu divided in five frames (Figure 6): 1.- Input files, 2.- Output files, 3.- Feature selection, and 4.- Feature parameters definition. 


\subsection{1.- Input files}

380

381

382

383

Image formats supported by FETEX 2.0 must be georeferenced and are those supported by ENVI (GeoTIFF, JPG2000, ENVI binary, ERDAS img, etc.). Limits of cartographic objects (parcels) must be contained in a ESRI shapefile spatial data format (.shp). In order to correctly superimpose both data, the image and the shapefile, they should be in the same spatial reference system.

FETEX 2.0 is able to work with large datasets of several images and shapefiles. In the case that a parcel (object) is represented along several image files, the program will internally build a mosaic and extract the final descriptive features from this new composed image.

\subsection{2.- Output files}

As a result of processing the image for object information extraction, an output table containing the values of the descriptive features selected (columns) for every object processed (rows) is obtained. This table can be available in four different formats: dBase, shapefile, ASCII and the format required by See5 software, which contains the C5.0 algorithm to generate decision trees.

In addition to the table with all the feature values needed for the classification of the objects, FETEX 2.0 provides the option of generating a set of screenshots and graphs that may be helpful for the interpretation of the results (Figure 7). The set of screenshots obtained for every object includes a color or grey level image of the object, the image of the wavelet details, image files of the GLCM of the original image and the wavelet details, the semivariogram graph, a binary image of the tree locations automatically detected, its Hough transform graph, and the binary image of the tree alignments. Additionally, a dbf file containing the semivariogram for each analyzed object can be generated.

\subsection{3.- Feature selection}

In this frame, the user can select the groups of features to be extracted from the objects. There are seven main groups of features: spectral, texture features based on the GLCM, wavelet 
derived texture features, descriptive parameters derived from the semivariogram, Hough transform based features, shape features, and qualitative features from a database.

406 On the right side of the frame, there are three drop-down lists for the selection of the image

407 bands from which the GLCM, wavelet and semivariogram features will be computed.

The last item in this menu enables us to use descriptive information from the input shapefile database as an additional descriptive feature. If the Database feature option button is enabled, the field of the shapefile database containing the descriptive feature must be selected from the adjoining drop-down list.

\subsection{4.- Feature parameters definition}

This frame is divided in three sections. In the first section, three general parameters of the process can be fixed: Minimum parcel size controls the minimum area of a polygon to be processed, avoiding very small polygons that may difficult a correct characterization. Parcel perimeter buffer is used to reject the peripheral pixels of the polygon in the analysis, in order to avoid the inclusion of pixels that are external to the object due to geo-referencing errors or misregistration between the database and the image. The values of the droplist represent the thickness of the rejecting buffer in pixels. NDVI bands droplist allows for the selection of the two image bands required for computing the NDVI: the IR and red bands.

The second section of this frame (Analysis options) allows for the selection of the specific features that can be obtained from the different feature groups, as well as the methodological parameters involved.

In the first tab, seven statistical features can be selected to be computed from each object. These features are computed for every band of the input image.

The second tab corresponds to the texture features. The number of grey levels to be considered in the computation of the GLCM can be chosen. The selection of this parameter is important, because the use of many grey levels does not necessarily mean an increase in the efficiency of 
429 the descriptors, but greatly increases the time consumed for computation. Furthermore, in 430 objects containing a low number of pixels, many grey levels in the computation of the GLCM 431 can make the characterisation of the distribution of the values in the matrix difficult.

432 The wavelet tab controls the wavelet function family and the support used to perform the 433 wavelet transform. Higher support values will require longer processing time. The number of 434 grey levels to be considered in the computation of the GLCM can also be chosen.

435 Two parameters can be controlled in the semivariogram tab: the maximum Semivariogram lag 436 size, and the Percentage of pixels from an object that are used to estimate its semivariogram, as 437 analyzed at the end of section 2.4.1.

438 In the Hough transform tab, several thresholds related to the automatic process to detect the 439 trees inside a parcel can be established. The Initial NDVI (parcel) parameter allows for the 440 definition of a threshold for the mean NDVI of the object. For those objects with a mean NDVI 441 higher than this value, the LMF method is applied using the NDVI image to locate the trees. On 442 the other hand, for the objects with a mean NDVI lower than this threshold value, the trees are located applying the LmF method using the IR band. Afterwards, two conditional thresholds must be established: those pixels located with the LMF method must have a NDVI value higher than the NDVI threshold to be accepted as a tree, whereas the IR band values of the pixels located with the LmF method must be lower than the IR band threshold.

447 Additionally, a selection of the threshold values used to define the size of the searching window 448 must be done, specifically the minimum and maximum Window diameter values. These values 449 must be in concordance with the minimum and maximum sizes of the trees, in pixels, that are 450 present in the specific geographic area.

451 The last tab corresponds to the selection of the shape features to be computed.

452 The last part of this frame (Attributes in the shapefile) first allows for the selection of the object identifier field in the shapefile of the database (ID), which must be an integer number. In 
addition to this, when a field containing the class of the training samples exists in the database, this can be selected in the last drop-down list (Samples). This information will be added at the end of the row of the feature vector corresponding to each object. The parcels that are not training samples will have a question mark at the end of the row. This field is required in order to obtain a See5 file format output.

\subsection{Future improvements}

FETEX 2.0 has been designed to work with large datasets, including images and shapefiles. In addition, some effort has been put to reduce the time needed to compute the different procedures used in the program, this is particularly important when a high number of objects must be processed.

The program allows for the extraction of a wide range of features from images and databases, some of them mainly focused on agricultural landscapes, with the final goal of describing the objects in depth in order to improve their classification. However, the program is intended to be a dynamic tool that progressively incorporates new feature extraction algorithms, as well as different types of spatial data which are currently more widely available,. The design and analysis of new descriptive features coming from different sources of information (airborne lidar systems, satellite radar images, etc.) will continue in order to advance in the description of objects. Useful three-dimensional information about the objects and the elements they may contain can be extracted from lidar data, complementing the current information available. In a different way, high resolution radar imagery can provide extra information about the roughness of the surfaces, which may complement the previous features. All these new sources of information are becoming more widely available to the user, and new tools to integrate and process the data will be needed. 
An object-oriented classification application example has been carried out using the descriptive

481 features extracted with FETEX 2.0. The test has been performed using data from a rural area in 482 the province of Castellón, on the Mediterranean coast of Spain. A series of aerial images

483 acquired in August 2005 with a Digital Mapping Camera (DMC) have been used, with a spatial resolution of $0.5 \mathrm{~m} /$ pixel and three spectral bands (green: $0.50-0.65 \mu \mathrm{m}$; red: $0.59-0.675 \mu \mathrm{m}$; and near infrared: $0.675-0.85 \mu \mathrm{m})$. A total of 616 training samples and 2438 evaluation samples have been selected from the cadastral polygons. The reference data used for evaluation have been obtained combining field work and photointerpretation. According to the type of landscape, ten different classes have been defined in the classification: Arable fields, Buildings, Carob-trees, Citrus orchards, Irrigated fields, Olive trees, Roads, Shrub-lands, and Young citrus orchards. Figure 8 shows some image-object examples of each class.

A set of different descriptive features has been computed using FETEX 2.0. They can be grouped as follows: (1) Spectral features: Mean, standard deviation, minimum and maximum from each band, and NDVI; (2) Texture features: GLCM derived features, edgeness factor, $1^{\text {st }}$ order descriptors, and wavelet based features computed from the red band; (3) Structural features: Hough transform and semivariogram derived features computed from the infrared band; and (4) Shape features.

Two classification methods have been used: Linear discriminant analysis (LDA), and decision trees computed using the C5.0 algorithm and combined with the boosting multi-classifier method. The classifications obtained have been evaluated by means of the error matrix (Congalton, 1991), from which the overall accuracy rate, the user's and producer's accuracies for every class have been computed.

Four tests per classification method have been done to independently evaluate the performance of each descriptive feature group. An additional classification has been performed combining all features. Table 2 shows the overall classification accuracies obtained using the different groups and combinations of descriptive features. In both classification methods, the use of all the 
506 features from the different groups together sharply increases the overall accuracy rate of the 507 classification. Additionally, no significant differences in overall accuracy are found between 508 both methods when all the feature groups are used, showing that the influence of the classifier is 509 not crucial when an exhaustive and complementary set of descriptive features is used. However, 510 the use of independent sets of features may introduce some differences in the overall accuracy 511 depending on the classification method used. Thus, using only spectral features, LDA provides 512 a better accuracy (65.5\%) than decision trees $(60 \%)$, but the latter increases the accuracy $513(62.1 \%)$ with respect to the LDA method (57.8\%) when only structural features are included for 514 classification. These differences may be due to the fact that the distribution of values of the two 515 groups of features, spectral and structural, are subject to specific ranges, and both classification 516 methods manage this information in different manner.

517 In order to study the influence or discriminant power that the variables (descriptive features) 518 have on the classification, two approaches have been employed: forward stepwise LDA and the 519 comparison of the percentage of use of each specific variable in all the decision trees created using the method of boosting. Figure 9 shows the per-class average of user's and producer's accuracies, and the overall classification accuracy when the 24 first features are progressively 522 included in the discriminant model. Figure 10 shows the percentage of use for the 24 descriptive 523 features most used by the classifier over the training objects.

524 The shape feature fractal dimension is the most frequently used in the decision trees and the 525 first discriminant feature selected in the stepwise LDA, allowing for the discrimination of class 526 Roads very efficiently, due to the characteristic long shape of the polygons that represent this 527 class. Particularly interesting is the fact that, always, some variables coming from the four 528 different groups considered are selected among the most relevant features. Using this short 529 group of variables the overall accuracy becomes stable, independently of the inclusion of 530 additional variables in the classification set (Figure 9). This illustrates again their 531 complementarity, as well as the possibility to increase the efficiency of the classification not 
532 only in terms of accuracy, but also in terms of reduction of the number of variables to be used,

533 by using only a selected group of features with low correlation.

\section{5.- SUMMARY}

A software program, FETEX 2.0, that extracts a set of descriptive features from image-objects and geodatabases is presented. A description of the features, classified into seven different groups and providing different and complementary information about the object, is carried out.

539 Some of them are particularly useful to characterize and classify agricultural landscapes and 540 have been presented for the first time here.

541 This software application generates an output table containing the object feature vectors 542 available in different formats, ready to use with different classifiers included in statistical 543 packages. Additionally, different types of descriptive graphs and images can be obtained to 544 facilitate the interpretation of processes and results. A classification example has been performed, showing the wide range of information obtained from each object. In this application example, it has been shown how the use of different types of variables provides a complete

547 description of the object, increasing the accuracy of the land use/land cover classification. The software program presented here allows for the computation of all these variables for their application to object-oriented classification, particularly interesting for agricultural mapping.

550 FETEX 2.0 can be considered to be a dynamic program in a continuous updating process in 551 order to incorporate new sources of data, new methods and features, and with the ability to be 552 oriented to specific applications. A limited version of FETEX 2.0 is available at the Geo553 Environmental Cartography and Remote Sensing Research Group (CGAT) website 554 (http://cgat.webs.upv.es). 
557 The authors appreciate the financial support provided by the Spanish Ministerio de Ciencia e 558 Innovación and the FEDER in the framework of the Project CGL2009-14220 and CGL2010559 19591/BTE, the Spanish Instituto Geográfico Nacional (IGN), Instituto Cartográfico 560 Valenciano (ICV), Instituto Murciano de Investigación y Desarrollo Agrario y Alimentario 561 (IMIDA) and Banco de Terras de Galicia (Bantegal).

\section{REFERENCES}

Aplin, P., Atkinson, P.M., Curran, P.J., 1999. Per-field classification of land use using the forthcoming very fine spatial resolution satellite sensors: problems and potential solution. In: Atkinson, P.M., Tate, P.M. (Eds.) Advances in Remote Sensing and GIS Analyses, Wiley \& Son, Chichester, pp. 219-239.

Ashoori, H., Fahimnejad, H., Alimohammadi, A., Soofbaf, S.R., 2008. Evaluation of the usefulness of texture measures for crop type classification by hyperion data. International Archives of the Photogrammetry, Remote Sensing and Spatial Information Sciences XXXVIIB8, 999-1006.

Baatz, M., Benz, U., Dehghani, S., Heynen, M., Höltje, A., Hofmann, P., Lingenfelder, I., Mimler, M., Sohlbach, M., Weber, M., Willhauck, G., 2004. eCognition USER GUIDE 4 on CD. of texture semivariogram features and their evaluation for object-oriented image classification. Computers \& Geosciences 36 (2), 231-240. remotely sensed images, In: De Jong, S.M., Van der Meer, F.D. (Eds.) Remote Sensing Image 
581 Berberoglu, S., Lloyd, C.D., Atkinson, P.M., Curran, P.J., 2000. The integration of spectral and 582 textural information using neural networks for land cover mapping in the Mediterranean. 583 Computers \& Geosciences 26 (4), 385-396.

584 Blaschke, T., 2010. Object based image analysis for remote sensing. ISPRS Journal of 585 Photogrammetry and Remote Sensing 65 (1), 2-16.

586 Bogaert, J., Rousseau, R., Hecke, P. V., Impens, I., 2000. Alternative area-perimeter ratios for 587 measurement of 2D shape compactness of habitats. Applied Mathematics and Computation 111 588 (1), 71-85.

589 Bultheel, A., 1995. Learning to swim in a sea of wavelets. Bulletin of the Belgian Mathematical 590 Society $2(1), 1-46$.

591 Carr, J.R., 1996. Spectral and textural classification of single and multiple band digital images. 592 Computers \& Geosciences 22(8), 849-865.

593 Carr, J.R., Miranda, F.P., 1998. The semivariogram in comparison to the co-occurrence matrix 594 for classification of image texture. IEEE Transactions on Geoscience and Remote Sensing 36 595 (6), 1945-1952.

596 Chanussot, J., Bas, P., Bombrun, L., 2005. Airborne Remote Sensing of Vineyards for the 597 Detection of Dead Vine Trees. In: Proceedings IEEE International Geoscience \& Remote 598 Sensing Symposium, Seoul, Korea, pp.3090- 3093.

599 Chica-Olmo, M., Abarca-Hernández,F., 2000. Computing geostatistical image texture for 600 remotely sensed data classification. Computers \& Geosciencies 26, 373-383.

601 Congalton, R., 1991. A review of assessing the accuracy of classications of remotely sensed 602 data. Remote Sensing of Environment 37 (1), 35-46. 
Debeir, O., Van den Steen, I., Latinne, P., Wolff, E., Van Ham, Ph., 2002. Spectral, spatial and contextual land cover classification using single and multiple classifiers. Photogrammetric Engineering \& Remote Sensing 68 (6), 597-605.

Durrieu, M., Ruiz, L.A., Balaguer, A., 2005. Analysis of geostatistical parameters for texture classification of satellite images. In: Proceedings EARSeL Symposium - Global Developments in Environmental Earth Observation from Space, Porto, Portugal, pp.11-18.

Fernández-Sarría, A., 2007. Estudio de técnicas basadas en la transformada wavelet y optimiación de sus parámetros para la clasificación por texturas de imágenes digitales. $\mathrm{PhD}$ Dissertation, Universidad Politécnica de Valencia, Spain, 247 pp.

612 Ferro, C.J., Warner, T.A., 2002. Scale and texture in digital image classification. 613 Photogrammetric Engineering \& Remote Sensing 68 (1), 51-63.

614 Gée, Ch., Bossu, J., Jones, G., Truchetet, F., 2008. Crop/weed discrimination in perspective agronomic images. Computers and Electronics in Agriculture 60 (1), 49-59.

616 Gougeon, F., 1999. Automatic individual tree crown delineation using a valley-following 617 algorithm and a rule-based system. In: Proceedings International Forum of Automated Interpretation of High Spatial Resolution Digital Imagery for Forestry, Victoria, BC, pp. 11-23. IEEE Transactions on Systems, Man and Cybernetics 3 (6), 610-622. and threats (SWOT). International Archives of the Photogrammetry, Remote Sensing and Spatial Information Sciences XXXVI - 4/C42 on CD.

624 Helmholz, P., Gerke, M., Heipke, C., 2007. Automatic discrimination of farmland types using 625 Ikonos imagery. In: Proceedings Photogrammetric Image Analysis 07, Munich, Germany, pp. $81-86$. 
627 Hermosilla, T., Díaz-Manso, J.M., Ruiz, L.A., Recio, J.A., Fernández-Sarría, A., Ferradáns-

628 Nogueira, P., 2010. Parcel-based image classification as a decision-making supporting tool for 629 the Land Bank of Galicia (Spain). International Archives of the Photogrammetry, Remote 630 Sensing and Spatial Information Sciences XXXVIII-4-8-2-W9, 40-45.

631 Hoffer, R.M., 1975. Natural resource mapping in mountainous terrain by computer analysis of 632 ERTS-1 Satellite Data. LARS Information Note 061575, Purdue University, Indiana, 124 pp.

633 Hough, P.V.C., 1962. Methods and means for recognizing complex patterns, U.S. Patent No. 6343069654.

635 Huang, X., Jensen, J.R., 1997. A machine-learning approach to automated knowledge-base 636 building for remote sensing image analysis with GIS data. Photogrammetric Engineering \& 637 Remote Sensing 63 (10), 1185-1194.

638 Hutchinson, C.F., 1982. Techniques for combining Landsat and ancillary data for digital 639 classification improvement. Photogrammetric Engineering \& Remote Sensing 48 (1), 123-130.

640 Jakomulska, A., Clarke, K.C., 2000. Variogram-derived measures of textural image 641 classification. Application to large scale vegetation mapping. In: Proceedings Third European 642 Conference on Geostatistics for Environmental Applications geoENV2000, Avignon, France, 643 pp. 181-202.

644 Janssen, L.L.F., Molenaar, M., 1995. Terrain objects, their dynamics and their monitoring by 645 the integration of GIS and remote sensing. IEEE Transactions on Geoscience and Remote $646 \quad$ Sensing $33(3), 749-758$.

647 Krummel, J. R., Gardner, R. H., Sugihara, G., O'Neill, V., Coleman, P. R., 1987. Landscape 648 patterns in a disturbed environment. OIKOS 48 (3), 321-324.

649 Leemans, V., Destain, M.-F., 2006. Application of the Hough transform for seed row 650 localisation using machine vision. Biosystems Engineering 94 (3), 325-336. 
651 Maillard, P., 2003. Comparing texture analysis methods through classification.

652 Photogrammetric Engineering \& Remote Sensing 69 (4), 357-367.

653 Mallat, S.G., 1989. A theory of multirresolution signal decomposition: The wavelet

654 representation. IEEE transactions on pattern analysis and machine intelligence 11 (7), 674-693.

655 Mas, J.F., 2003. An artificial neural networks approach to map land use/cover using Landsat 656 imagery and ancillary data. In: Proceedings IEEE International Geosciences \& Remote Sensing 657 Symposium, Toulouse, France, pp. 3498-3500.

658 McGarigal, K., Marks, B. J., 1995. FRAGSTATS: spatial pattern analysis program for 659 quantifying landscape structure. General Technical Report PNW-GTR-351. Portland, OR: U.S. 660 Department of Agriculture, Forest Service, Pacific Northwest Research Station. 122 pp.

661 Meinel, G., Neubert, M., 2004. A comparison of segmentation programs for high resolution 662 remote sensing data. International Archives of the Photogrammetry, Remote Sensing and 663 Spatial Information Sciences XXXV-B4, 1097-1102.

664 Neubert, M., Herold, H., 2008. Assessment of remote sensing image segmentation quality. 665 International Archives of the Photogrammetry, Remote Sensing and Spatial Information 666 Sciences XXXVIII-4/C1 on CD.

667 Pedley, M.I., Curran, P.J., 1991. Per-field classification: an example using SPOT-HRV imagery. 668 International Journal of Remote Sensing 12 (11), 2181-2192.

669 Pyrcz, M. J., Deutsch, C. V., 2003. The whole story on the hole effect. In: Searston, S. (Eds.) 670 Geostatistical Association of Australasia Newsletter 18.

671 Randen, T., Husoy, J.H., 1999. Filtering for texture classification: A comparative study. IEEE 672 Transactions on Pattern Analysis and Machine Intelligence 21 (4), 291-310. 
Recio, J., 2009. Técnicas de extracción de características y clasificación de imágenes orientada a objetos aplicadas a la actualización de bases de datos de ocupación del suelo. PhD Dissertation. Universidad Politécnica de Valencia, Spain, 289 pp.

Recio, J.A., Hermosilla, T., Ruiz, L.A., Fernández-Sarría, A., 2009. Analysis of the addition of qualitative ancillary data on parcel-based image classification. International Archives of the Photogrammetry, Remote Sensing and Spatial Information Sciences XXXVIII-1-4-7/W5 on CD.

Recio, J.A., Ruiz, L.A., Fernández-Sarría, A., Hermosilla, T., 2006. Integration of multiple feature extraction and object oriented classification of aerial images for map updating. In: Proceedings Second International Symposium on Recent Advances in Quantitative Remote Sensing, Torrent, Spain, pp. 391-396.

Recio, J.A., Hermosilla, T., Ruiz, L.A., Fernández-Sarría, A., 2010. Addition of geographic ancillary data for updating geo-spatial databases. International Archives of the Photogrammetry, Remote Sensing and Spatial Information Sciences XXXVIII-4-8-2-W9, 46-51.

Reid, J.F., Searcy, S.W., 1986. Detecting crop rows using the Hough transform. ASAE Paper No. 86-3042, St. Joseph, MI, USA.

Rogan, J., Miller, J., Stow, D., Frankling, J., Levien, L., Fischer, C., 2003. Land cover change mapping in California using classification trees with multitemporal Landsat TM and ancillary data. Photogrammetric Engineering \& Remote Sensing 69 (7), 793-804.

Ruiz, L.A., Fernández-Sarría, A., Recio, J, 2002. Evaluation of texture analisis techniques to characterize vegetation. In: Proceedings International Symposium on Recent Advances in Quantitative Remote Sensing, Torrent, Spain, pp. 514-521.

Ruiz, L.A, Fernández-Sarría, A., Recio, J.A., 2004. Texture feature extraction for classification of remote sensing data using wavelet decomposition: A comparative study. International 

B4, 1109-1114.

Ruiz, L.A., Recio, J.A., Hermosilla, T., 2007. Methods for automatic extraction of regularity patterns and its application to object-oriented image classification. International Archives of the Photogrammetry, Remote Sensing and Spatial Information Sciences XXXVI-3/W49A, 117-121.

Ruiz, L.A., Recio, J.A, Hermosilla, T., Fernández-Sarría, A., 2009. Identification of agricultural and land cover database changes using object-oriented classification techniques. In: Proceedings 33rd International Symposium on Remote Sensing of Environment, Stresa, Italy, on CD. construction of multiscale texture maps of SAR images. Canadian Journal of Remote Sensing $24(3), 264-285$.

Smith, G., Morton, D., 2008. Segmentation: The Achilles heel of object-based image analysis?. International Archives of the Photogrammetry, Remote Sensing and Spatial Information Sciences, XXXVIII-4/C1 on CD. disease. IEEE Transactions on Computers 21 (7), 667-676.

714 Trías-Sanz, R., 2006. Texture orientation and period estimation for discriminating between 715 forests, orchards, vineyards, and tilled filled. IEEE Transactions on Geoscience and Remote 716 Sensing 44 (10), 2755-2760.

717 Van Coillie F.M.B., Pires, P.L.V.M., Van Camp, N.A.F, Gautama, S., 2008. Quantitative 718 segmentation evaluation for large scale mapping purposes. International Archives of the 719 Photogrammetry, Remote Sensing and Spatial Information Sciences, XXXVIII-4/C1 on CD. 
720 Van de Wouwer, G., Scheunders, P., Van Dyck, D., 1999. Statistical texture characterization

721 from discrete wavelet representations. IEEE Transactions on Image Processing 8 (4), 592-598.

722 Volante, J. N., Campos, C. J., Noé, Y. E., Elena, H. J., 2007. Método de clasificación "por 723 parcela" para la detección de cultivos: Aplicación al área agrícola de Las Lajitas (Salta, 724 Argentina), (Per-field classification method to detect crops: Application in agricultural area of 725 Las Lajitas (Salta, Argentina)). In: Proceedings XII Congress Remote Sensing Spanish 726 Association, Mar del Plata, Argentina, pp. 79-86.

727 Walker, J.S.,1999. A Primer on Wavelets and their Scientific Applications, 1st edn., Chapman 728 \& Hall/CRC, Boca Raton, FL, 155 pp.

729 Walter, V., 2004. Object-based classification of remote sensing data for change detection. 730 ISPRS Journal of Photogrammetry and Remote Sensing 58 (3-4), 225-238.

731 Woodcock, C.E., Strahler, A.H., Jupp, D.L.B., 1988a. The use of variograms in remote sensing

732 I: Scene models and simulated images. Remote Sensing of Environment 25 (3), 323-348.

733 Woodcock, C.E., Strahler, A.H., Jupp, D.L.B., 1988b. The use of variograms in remote sensing 734 II: real digital images. Remote Sensing of Environment 25 (3), 349-379.

735 Wulder, M., Niemann, K.O., Goodenough, G.D., 2000. Local maximum filtering for the 736 extraction of tree locations and basal area from high spatial resolution imagery. Remote Sensing 737 of Environment 73 (1), 103-114.

738 Zhang, H., Fritts, J.E., Goldman, S.A., 2008. Image segmentation evaluation: A survey of 739 unsupervised methods. Computer Vision and Image Understanding 2 (110), 260-280. 


\begin{tabular}{|c|c|c|c|}
\hline \multicolumn{2}{|c|}{ Spectral Features (number indicates the band) } & \multicolumn{2}{|c|}{ Hough transform features } \\
\hline MEAN1 & Mean value of band 1 & ANG_DIF & Angular difference between the two principal directions \\
\hline STDEV1 & Standard deviation value of band 1 & ANG1PERC & Proportion of straight lines in the principal direction \\
\hline MIN1 & Minimum value of band 1 & ANG2PERC & Proportion of straight lines in the secondary direction \\
\hline MAX1 & Maximum value of band 1 & RHO1MEAN & $\begin{array}{l}\text { Mean of the distances between straight lines in the } \\
\text { principal direction }\end{array}$ \\
\hline RANGE1 & Range of values of band 1 & RHO1 STDEV & $\begin{array}{l}\text { Standard deviation of the distances between straight } \\
\text { lines in the principal direction. }\end{array}$ \\
\hline SUM1 & Summatory of values of band 1 & PT1PERC & Proportion of points included in the principal direction \\
\hline MAJORITY1 & Mode of values of band 1 & PT1NORM & $\begin{array}{l}\text { Proportion of points included in the principal direction } \\
\text { normalized by area }\end{array}$ \\
\hline MEANNDVI & Mean value of NDVI & RHO1MEDIAN & $\begin{array}{l}\text { Median of the distances between straight lines in the } \\
\text { principal direction }\end{array}$ \\
\hline STDEVNDVI & Standard deviation value of NDVI & RHO2MEAN & $\begin{array}{l}\text { Mean of the distances between straight lines in the } \\
\text { secondary direction }\end{array}$ \\
\hline MINNDVI & Minimum value of NDVI & RHO2 STDEV & $\begin{array}{l}\text { Standard deviation of the distances between straight } \\
\text { lines in the secondary direction. }\end{array}$ \\
\hline MAXNDVI & Maximum value of NDVI & PT2PERC & Proportion of points included in the secondary direction \\
\hline RANGENDVI & Range of values of NDVI & PT2NORM & $\begin{array}{l}\text { Proportion of points included in the secondary direction } \\
\text { normalized by area }\end{array}$ \\
\hline SUMNDVI & Summatory of values of NDVI & RHO2MEDIAN & $\begin{array}{l}\text { Median of the distances between straight lines in the } \\
\text { secondary direction }\end{array}$ \\
\hline \multicolumn{2}{|l|}{ Texture Features } & \multicolumn{2}{|c|}{ Semivariogram based features } \\
\hline MEAN_EDG & Mean value of edgeness factor & RVF & $\begin{array}{l}\text { Ratio between the values of the total variance and the } \\
\text { semivariance at first lag }\end{array}$ \\
\hline STDEV_EDG & Standard deviation of edgeness factor & RSF & $\begin{array}{l}\text { Ratio between semivariance values at second and first } \\
\text { lag }\end{array}$ \\
\hline UNIFOR & GLCM Uniformity & FDO & First derivative near the origin \\
\hline ENTROP & GLCM Entropy & FML & First maximum lag value \\
\hline CONTRAS & GLCM Contrast & MFM & $\begin{array}{l}\text { Mean of the semivariogram values up to the first } \\
\text { maximum }\end{array}$ \\
\hline IDM & GLCM Inverse Difference Moment & VFM & $\begin{array}{l}\text { Variance of the semivariogram values up to the first } \\
\text { maximum }\end{array}$ \\
\hline COVAR & GLCM Covariance & RMM & $\begin{array}{l}\text { Ratio between the semivariance at first local maximum } \\
\text { and the mean semivariogram values up to this } \\
\text { maximum }\end{array}$ \\
\hline VARIAN & GLCM Variance & DMM & $\begin{array}{l}\text { Distance between the first maximum and the first } \\
\text { minimum }\end{array}$ \\
\hline CORRELAT & GLCM Correlation & \multicolumn{2}{|l|}{ Shape features } \\
\hline SKEWNESS & Skewness value of the histogram & COMPACT & Compactness \\
\hline KURTOSIS & Kurtosis value of the histogram & SH_INDEX & Shape Index \\
\hline \multicolumn{2}{|c|}{ Wavelet based features } & FRACTAL & Fractal dimension \\
\hline MEAN_W1 & GLCM Mean of the details image & AREA & Area \\
\hline UNIFOR_W1 & GLCM Uniformity of the details image & PERIMETER & Perimeter \\
\hline ENTROP_W1 & GLCM Entropy of the details image & \multicolumn{2}{|c|}{ Ancillary data features } \\
\hline CONTRAS_W1 & GLCM Contrast of the details image & ANCILLARY & Ancillary data from database \\
\hline IDM_W1 & $\begin{array}{l}\text { GLCM Inverse Difference Moment of the details } \\
\text { image }\end{array}$ & \multicolumn{2}{|c|}{ Processing information } \\
\hline COVAR_W1 & GLCM Covariance of the details image & PROCESSED & Processing information \\
\hline VARIAN_W1 & GLCM Variance of the details image & \multicolumn{2}{|c|}{ Training sample identification } \\
\hline CORRELAT_W1 & GLCM Correlation of the details image & SAMPLE & Training sample class \\
\hline MEAN_EDG_W1 & Mean value of edgeness factor of the details image & & \\
\hline STDEV_EDG_W1 & $\begin{array}{l}\text { Standard deviation of edgeness factor of the } \\
\text { details image }\end{array}$ & & \\
\hline
\end{tabular}




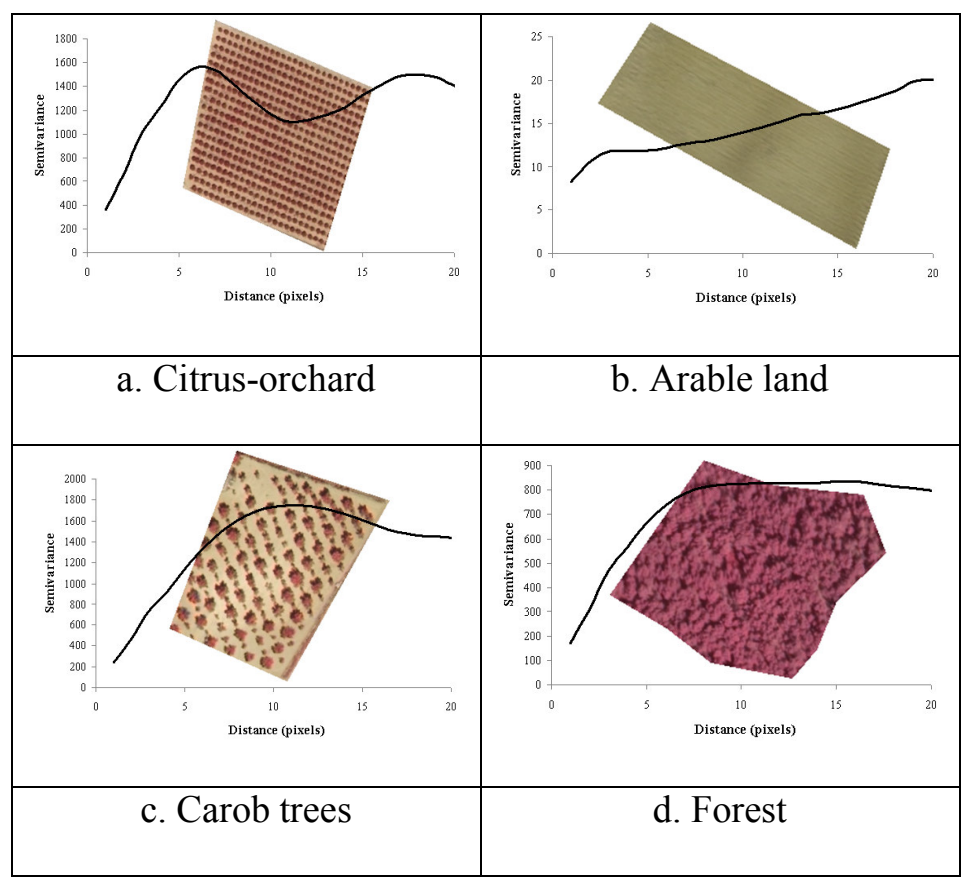

743 Figure 1. Parcels with different land use and their respective experimental semivariogram superimposed. Distance in pixels is in abscissas, and semivariance values are represented in ordinates. 


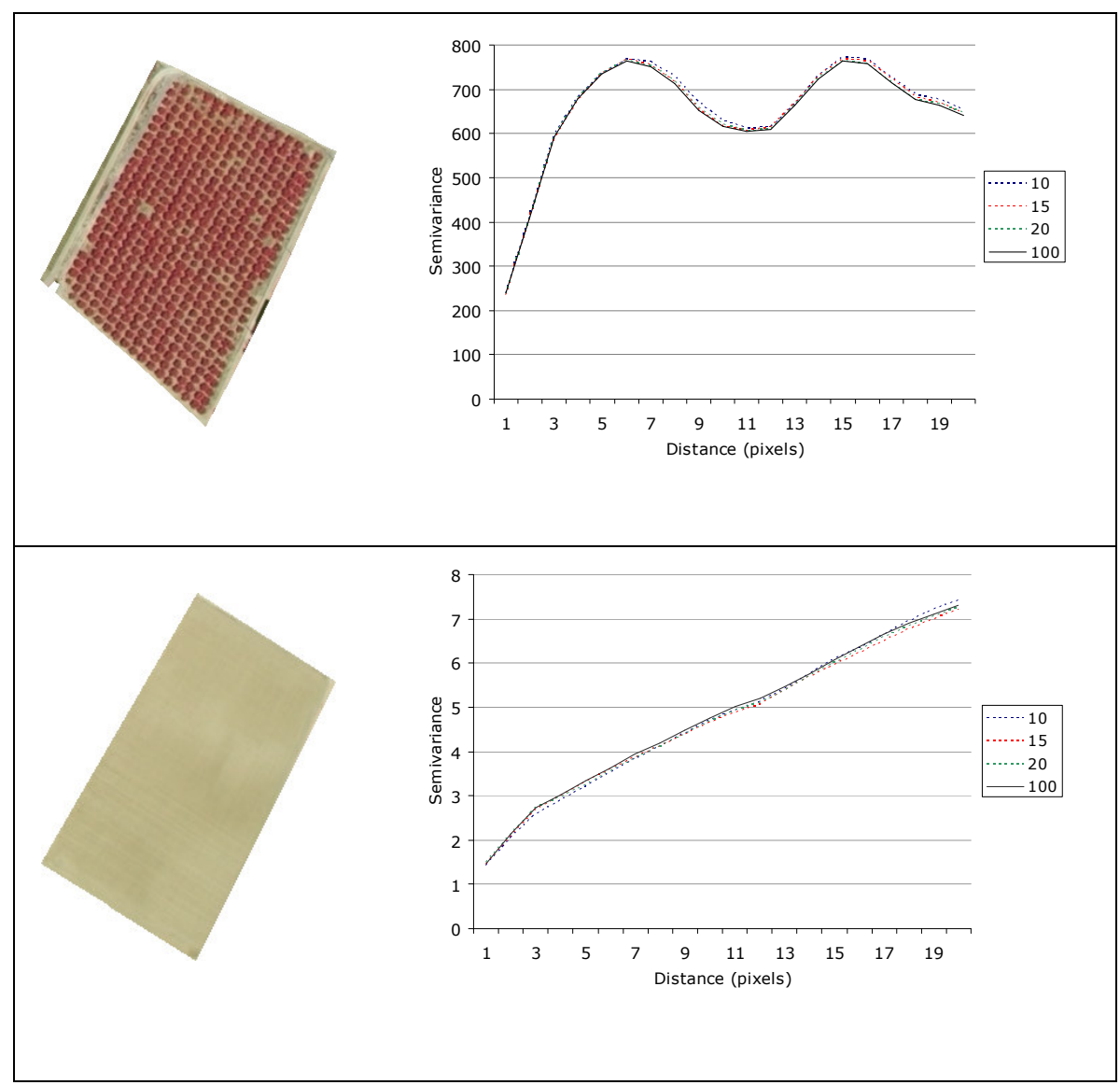

747 Figure 2. Semivariograms computed using different pixel percentages (10, 15 and 20) compared to semivariograms computed using all pixels in object. 


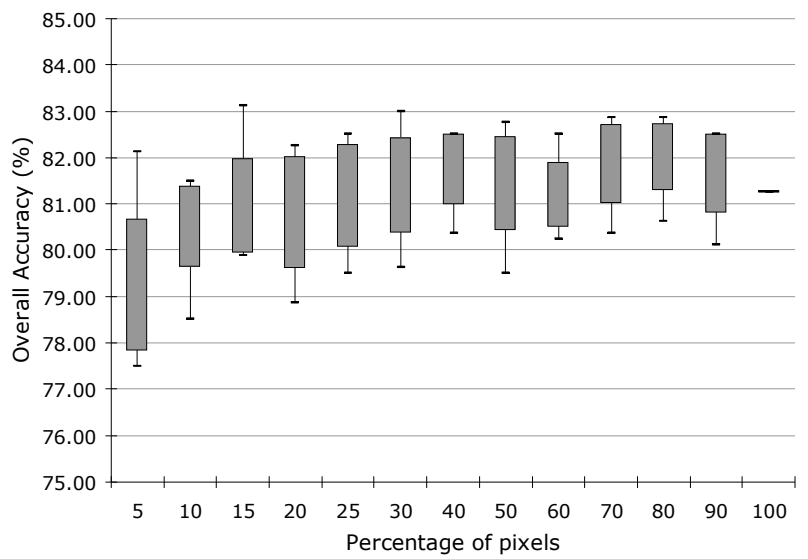

Figure 3. Box and whiskers graph representing overall accuracies of a series of 10

752 classifications for each pixel percentage used to compute semivariogram. Whiskers represent maximum and minimum values; boxes represent one standard deviation apart from mean values. 


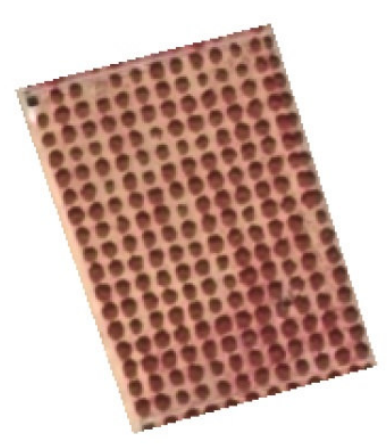

a.

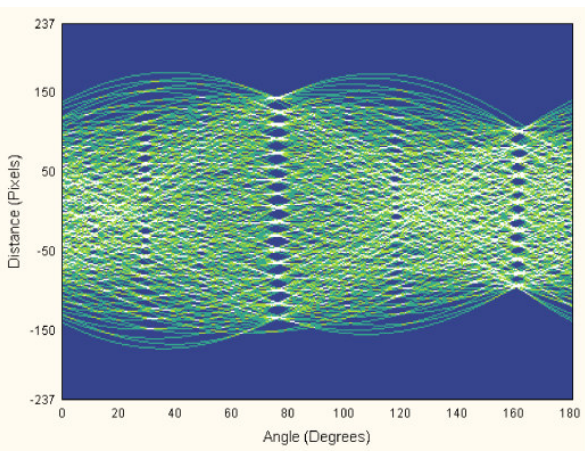

c.

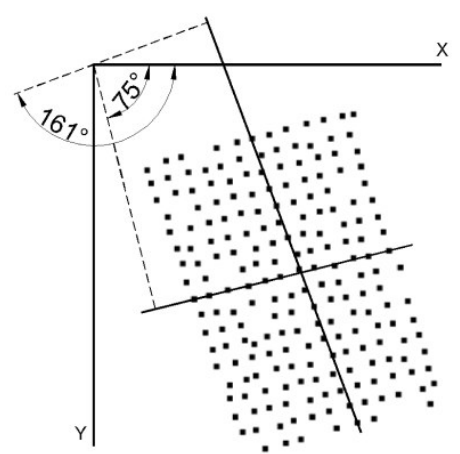

b.

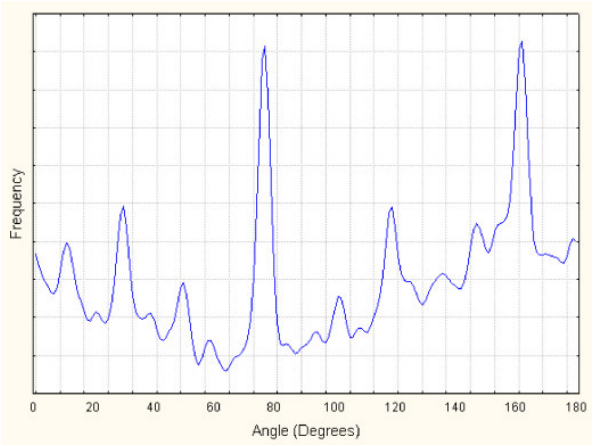

d

756 Figure 4. Example of application of the Hough transform method: (a) object in image space; (b)

757 local maxima detection; (c) Hough transform space; (d) histogram of coincidences in different directions. Main directions are extracted at $75^{\circ}$ and $161^{\circ}$. 


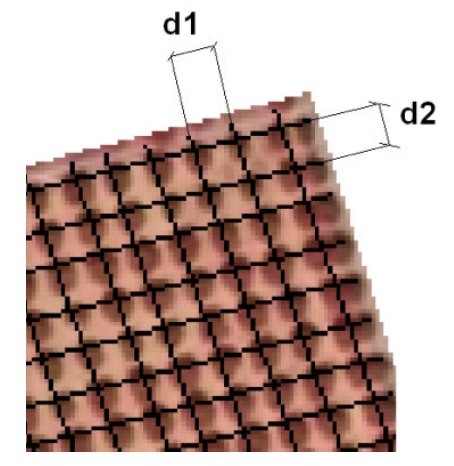

Figure 5. Extraction of planting pattern distances. 


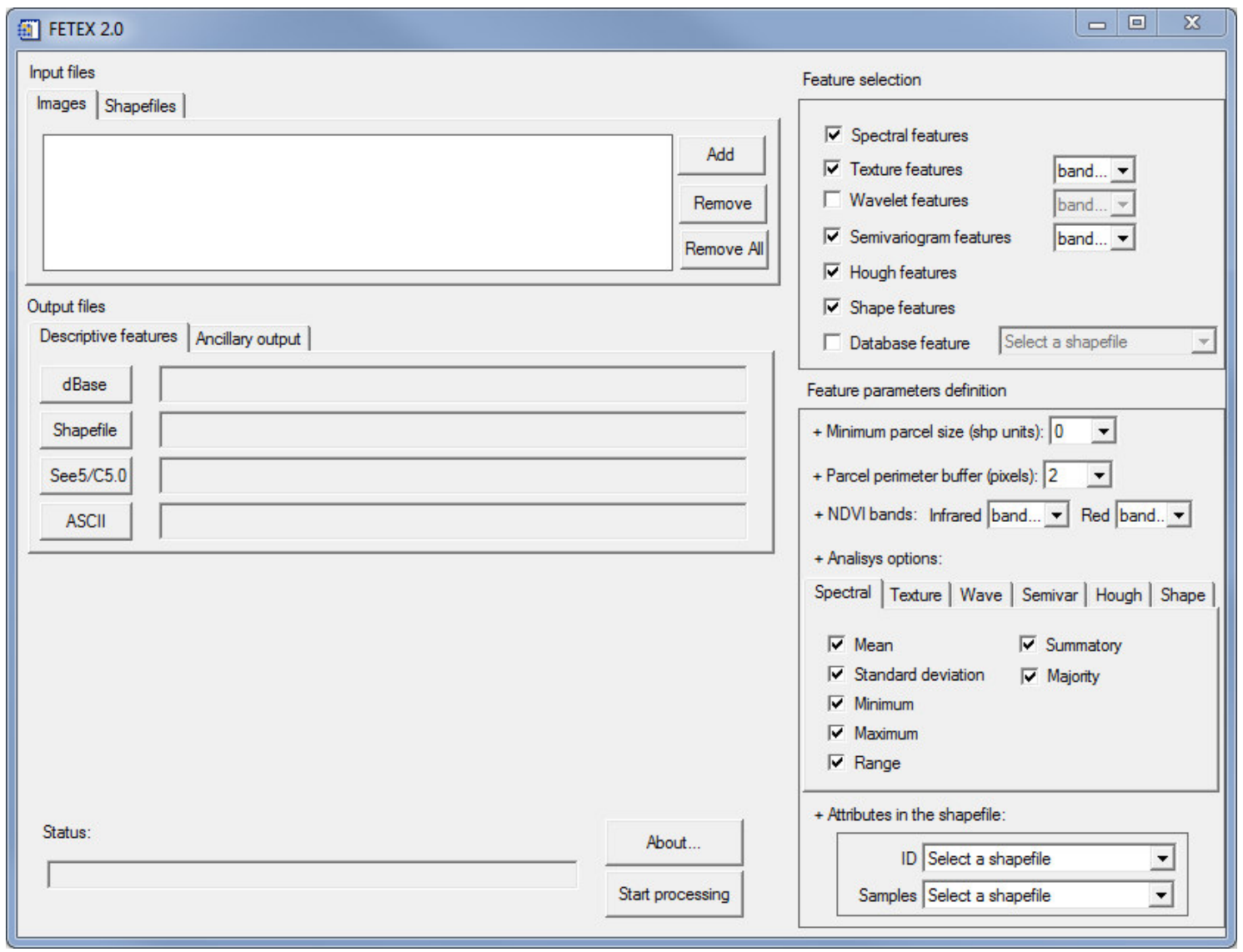

Figure 6. Graphic user interface of FETEX 2.0. 


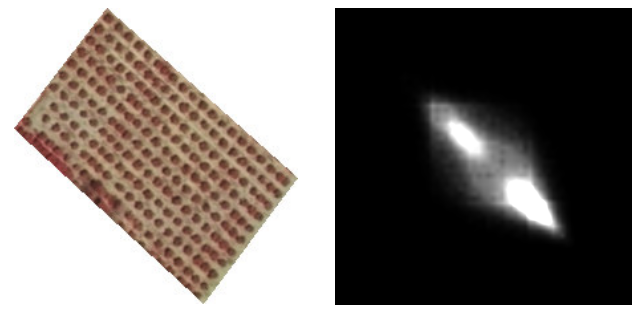

a

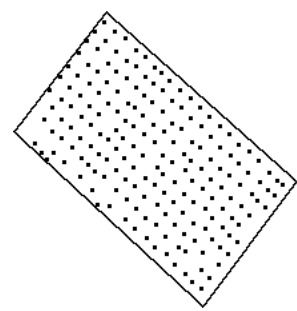

$\mathrm{f}$

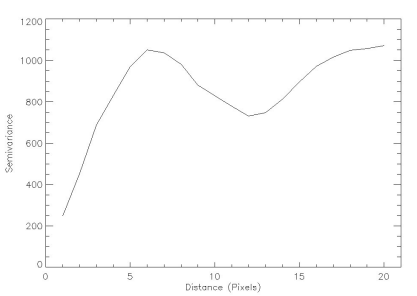

$\mathrm{c}$

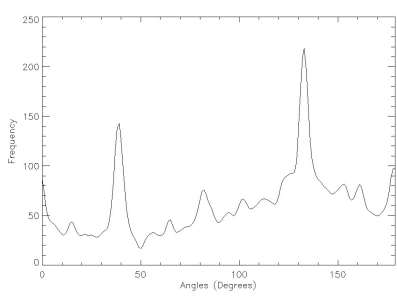

h
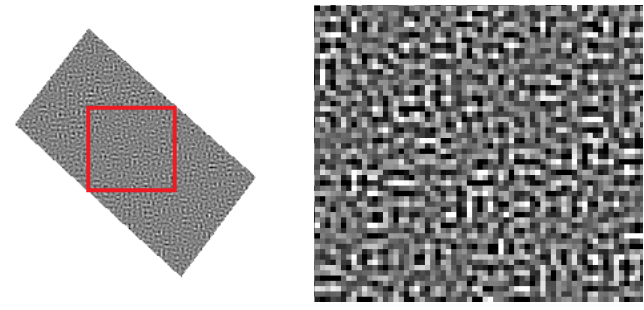

d
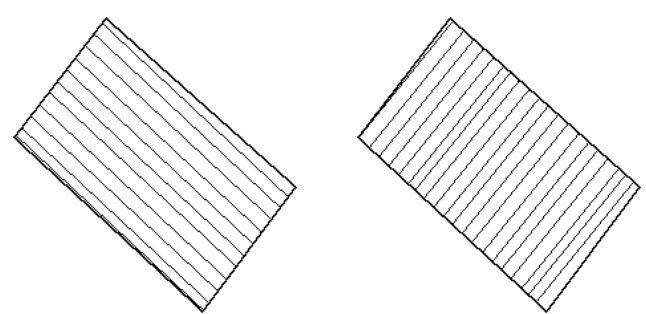

$\mathrm{j}$

Figure 7. Examples of screenshots generated by FETEX 2.0 in a citrus crop parcel: (a) Color infrared image of object; (b) grey level image representation of the GLCM; (c) semivariogram graph (with hole-effect presence); (d) sum of details of wavelet decomposition (Coiflet, size 24 pixels); (e) detail of (d); (f) local maxima detected representing trees; (g) Hough space representation of local maxima; (h) Hough transform directions histogram; (i) alignments in main direction; (j) alignments in second direction. 

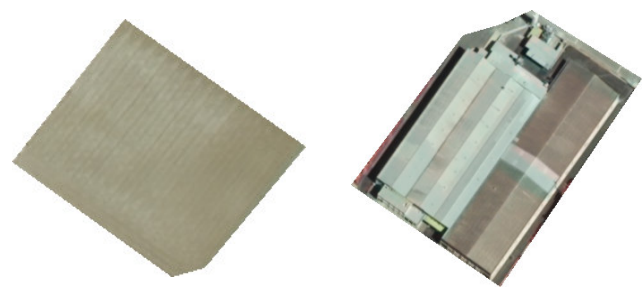

Arable fields

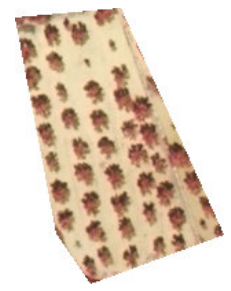

Olive trees
Buildings

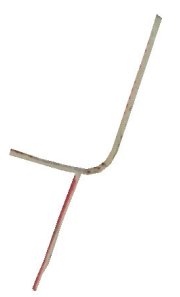

Roads

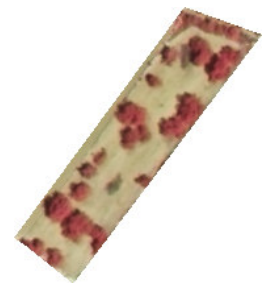

Carob-trees

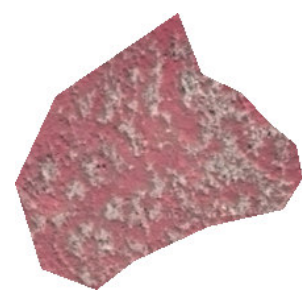

Shrub-lands
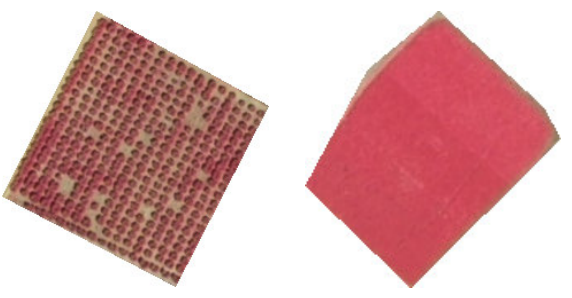

Citrus orchards

Irrigated fields

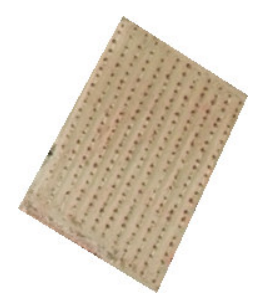

\section{Young citrus}

orchards 

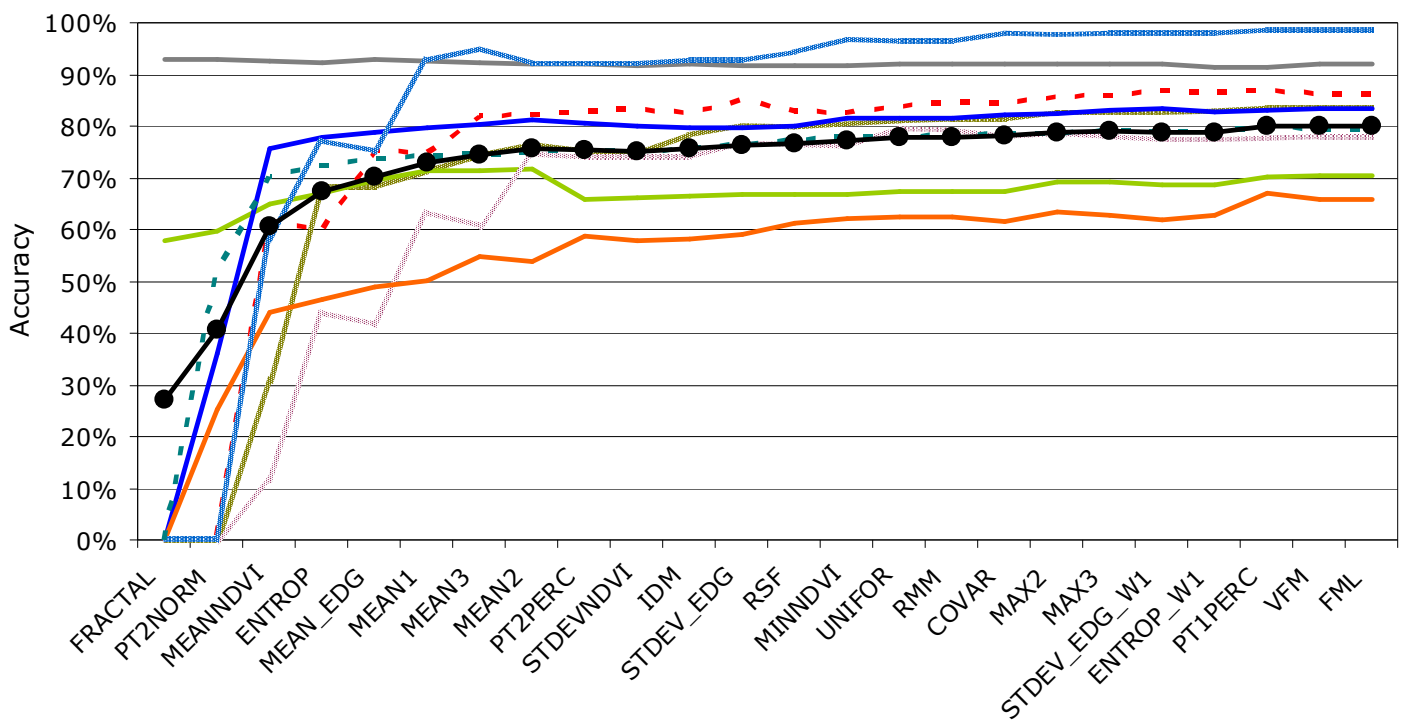

Variables in the discriminant model

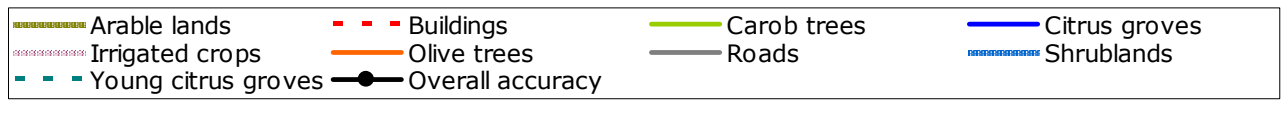

Figure 9.- Overall classification accuracy and per-class accuracies when new variables are 


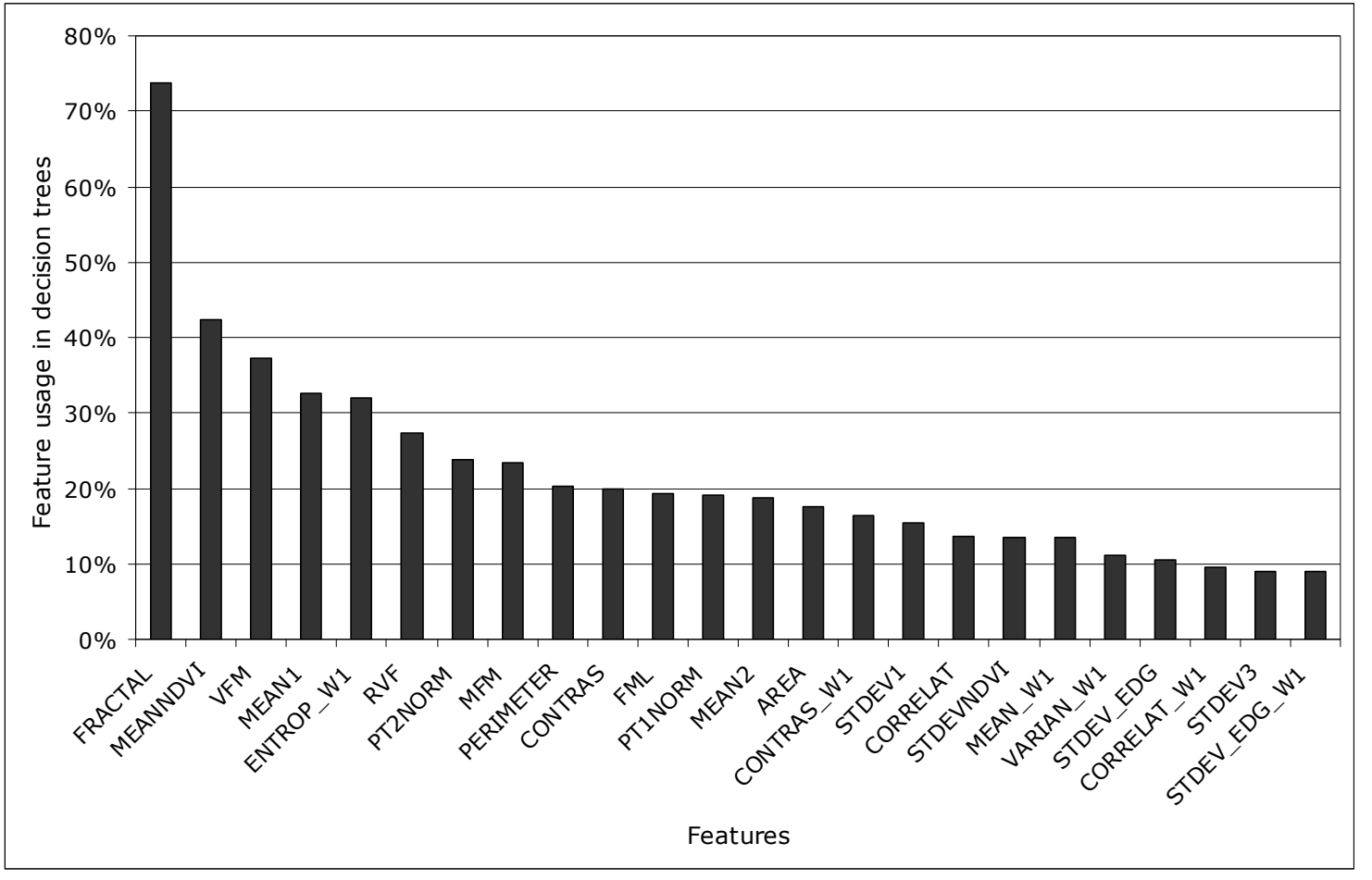

Figure 10.- Feature percentage of use in decision tree construction. 
Table 1.-Shape parameters computed in FETEX 2.0.

$$
C=\frac{4 \cdot \pi \cdot \text { Area }^{2}}{\text { Perimeter }^{2}} \quad S I=\frac{\text { Perimeter }}{4 \cdot \sqrt{\text { Area }}} \quad F D=2 \cdot \frac{\log \left(\frac{\text { Perimeter }}{4}\right)}{\log (\text { Area })}
$$
a. Compactness
b. Shape index
c. Fractal dimension 


\begin{tabular}{|l|c|c|}
\cline { 2 - 3 } \multicolumn{1}{c|}{} & \multicolumn{2}{c|}{ Classification method } \\
\hline Descriptive feature groups & $\begin{array}{c}\text { Linear } \\
\text { discriminant } \\
\text { analysis }\end{array}$ & $\begin{array}{c}\text { Decision trees } \\
\text { (C5.0) }\end{array}$ \\
\hline Spectral features & $65.5 \%$ & $60.0 \%$ \\
\hline Texture features & $65.4 \%$ & $66.7 \%$ \\
\hline Structural features & $57.8 \%$ & $62.1 \%$ \\
\hline Shape features & $32.2 \%$ & $35.4 \%$ \\
\hline All features & $\mathbf{8 1 . 7 \%}$ & $\mathbf{8 0 . 6 \%}$ \\
\hline
\end{tabular}

